\title{
Mitochondrial Fission Regulator 2 (MTFR2) Confers anti-ER Endocrine Therapy Resistance to Breast Cancer Cells by Modulating Mitochondrial Autophagy through Direct Phosphorylation of FUNDC1
}

\section{Zhanqiang Zhang}

Sun Yat-sen University First Affiliated Hospital

Guanming Lu

Youjiang Medical University for Nationalities

\section{Bo Lin}

Sun Yat-sen University First Affiliated Hospital

\section{Yanghong Li}

Youjiang Medical University for Nationalities

\section{Fuju Li}

Youjiang Medical University for Nationalities

\section{Zipeng Wen}

Youjiang Medical University for Nationalities

\section{Weiming LV}

Sun Yat-sen University First Affiliated Hospital

\section{Yuanhui Lai}

Sun Yat-sen University First Affiliated Hospital

jie li ( $\square$ lijie78@mail.sysu.edu.cn )

Sun Yat-sen University First Affiliated Hospital https://orcid.org/0000-0003-1232-3248

Research article

Keywords: MTFR2, breast cancer, endocrine -therapy resistance, autophagy

Posted Date: August 3rd, 2021

DOl: https://doi.org/10.21203/rs.3.rs-757543/v1

License: (c) (i) This work is licensed under a Creative Commons Attribution 4.0 International License. Read Full License 


\section{Abstract}

Background: Mitochondrial fission regulator 2 (MTFR2) belongs to the MTFR1/ family with sequence similarity 54 (FAM54) family. Recently, it was reported that MTFR2 promotes the proliferation and invasion of breast cancers (BCs). However, the relationship between MTFR2 and endocrine crine therapy resistance is still unknown.

Materials and methods: We collected $36 \mathrm{ER}+\mathrm{BC}$ tissues and adjacent normal tissues and 10 samples from patients who received endocrine therapy. We detected the expression pattern of MTFR2 and the fold change in MTFR2 in cells treated with tamoxifen and letrozole. The autophagy status was also determined.

Results: MTFR2 expression was upregulated in $\mathrm{ER}+\mathrm{BC}$ tissues and was strongly upregulated in the samples that were treated with endocrine therapy. Knocking out MTFR2 increased BC cell sensitivity to endocrine therapy. In addition, MTFR2 directly bound to FUNDC1 and promoted FUNDC1 phosphorylation, thus inhibiting autophagy.

Conclusion: Taken together, our results indicate that increased expression of MTFR2 is associated with endocrine therapy resistance in BC cells. Our findings indicate that MTFR2 could serve as a novel therapeutic target for $\mathrm{ER}+\mathrm{BC}$ patients who suffer from endocrine therapy resistance.

\section{Introduction}

Breast cancer $(\mathrm{BC})$ is one of the most common cancers worldwide and the second leading cause of cancer-related mortality in women(1). Among all cancers, approximately $70 \%$ of patients are ER+(2). With the advantages of individualized therapy in recent decades, patients benefit from anti-ER therapy, also called endocrine therapy. However, not all patients are candidates for endocrine therapy, and a certain proportion of patients still suffer from endocrine therapy resistance(3). Exploring the mechanism of endocrine therapy resistance is one of the hottest fields in the world, and many contributions have been made to this topic in recent years. LEM4 activates the CDK-Rb pathway and thus helps BC cells escape tamoxifen(4). PIK3CA, AKT1 and ESR1 mutations were also detected in tamoxifen-resistant cell lines(5). The loss of NF1 function was reported to contribute to tamoxifen resistance(6).

Autophagy is a critical physiological program in which cells collect misfolded proteins and injured organelles and send them to lysosomes for recycling(7). The dysregulation of autophagy was reported to be involved in the tumorigenesis of cancers such as gastric cancer(8), colon cancer(9) and glioma(10). Targeted therapy focusing on the autophagy pathway is one of the hottest fields in the world and has attracted increasing attention. Autophagy is involved in the tumorigenesis and progression of $\mathrm{BC}(11)$. It has been reported that alterations in autophagy status promote proliferation, migration and invasion and helps cancer cells escape chemotherapy and endocrine therapy. Autophagy is mediated by various pathways, such as the well-known autophagy-associated genes (ATGs), transcription factors such as 
FOXM1(12) and other metabolic enzymes such as LDHA(13). However, the exact mechanism by which autophagy is altered in $\mathrm{BC}$ is still unknown.

Mitochondrial fission regulator 2 (MTFR2), also called family with sequence similarity 54 , member A (FAM54A), has been poorly studied in tumors. Recent research has demonstrated that MTFR2 promotes proliferation, migration and invasion by switching glucose metabolism from OXPHOS to glycolysis(14). However, the function and underlying mechanism of MTFR2 in endocrine therapy resistance are still unknown.

In this study, we aimed to uncover the relationship between MTFR2 and endocrine therapy resistance. Our results demonstrate for the first time that MTFR2 is upregulated in ER + BC tumors and that MTFR2 could help cancer cells acquire endocrine therapy resistance.

\section{Results}

\section{MTFR2 is upregulated in ER + BC tissues and confers tamoxifen and letrozole resistance to $\mathrm{BC}$ cells.}

To examine MTFR2 expression in ER + BC, we collected 30 paired $E R+B C$ tumors and adjacent normal tissues and analyzed the relative MTFR2 mRNA level. The data are shown in Fig. 1A. MTFR2 expression was upregulated in tumors compared with normal tissues $(p<0.001)$. We next randomly chose 8 tumor tissues and paired normal tissues and utilized immunoblotting to detect the protein level of MTFR2. The results showed that MTFR2 expression was upregulated in 6 out of 8 patients. To explore the potential function of MTFR2 in endocrine therapy resistance, we collected 10 tumor tissues before and after standard endocrine therapy and analyzed the mRNA and protein levels of MTFR2. MTFR2 expression was upregulated in the tissues after endocrine therapy at both the mRNA and protein levels (Fig. 1C, 1D, p $<0.001$ ). We next treated ER + BC cells (MCF-7 and T47D cells) with tamoxifen (TMX) and letrozole (Let) and detected the expression pattern of MTFR2. The results showed that TMX and Let treatment upregulated MTFR2 expression at both the RNA and protein levels. (Fig. 1E, 1F, p < 0.001). We next applied KEGG pathway analysis and found that MTFR2 expression was strongly correlated with proliferation and apoptosis, indicating that MTFR2 may be engaged in endocrine therapy resistance, which was consistent with previous results (Fig. 1G).

\section{MTFR2 promotes endocrine therapy resistance in BC cells.}

To uncover the exact function of MTFR2 in the endocrine therapy resistance of ER + BC cells, we established stable MTFR2-knockout (KO) cell lines with CRISPR-Cas9. After treatment with TMX and Let, cell viability and proliferation status were examined. Both cell viability (Fig. $2 A, p<0.001)$ and long-term proliferation after treatment with TMX or Let decreased in KO cell lines compared with control cell lines (Fig. 2B, p < 0.001). Apoptosis rates were detected with flow cytometry, and stable MTFR2-KO cell lines 
exhibited increased levels of apoptosis after treatment with TMX and Let (Fig. $2 \mathrm{C}, \mathrm{p}<0.001)$. We next detected apoptosis markers, such as the caspase cascade. Cleaved caspase, the active form of caspase, increased with in MTFR2-KO cells after treatment with TMX and Let (Fig. 2D, $p<0.001$ ).

\section{Knocking out MTFR2 increases autophagic flux in BC cells.}

Autophagy is reported to be involved in chemotherapy resistance in other cancers(15). We thus detected autophagy status, and the ratio of LC3B-II to LC3B-I increased with MTFR2 KO (Fig. 3A). We next utilized immunofluorescence to detect LC3 puncta. The number of LC3 puncta per cell in MTFR2-KO cells was increased compared with that in the control (Fig. 3B, 3C, p < 0.001). However, the accumulation of LC3B puncta was due to an increase in the production of LC3B-II or a decrease in the degradation of p62. We next detected the level of p62, and the results are shown in Fig. 3D. p62 expression increased in MTFR2$\mathrm{KO}$ cell lines. Taken together, these results suggest that KO of MTFR2 promotes autophagic flux and thus is potentially responsible for acquired resistance.

\section{MTFR2 inhibits mitochondrial autophagy by directly binding to FUNDC1.}

MTFR2 is a member of the mitochondrial fission regulator family and is primarily located on mitochondria. We hypothesized that the change in autophagy status was caused by the dysregulation of mitochondrial autophagy. We next detected markers such as PINK and FUNDC1, and neither of them showed any expression difference in the different cell lines (data not shown). We next utilized immunoprecipitation in cells transfected with HA-MTFR2 and Flag-FUNDC1 and found that MTFR2 directly binds to FUNDC1 (Fig. 4A). Immunofluorescence results also showed that MTFR2 colocalized

with FUNDC1 in the cytoplasm (Fig. 4B). We next detected the protein level of FUNDC1 and found that the total level of FUNDC1 was unchanged, while the level of phosphorylated FUNDC1 was decreased in the MTFR2-KO cell lines after treatment with TMX and Let (Fig. 4C). For further conformation, we reexpressed MTFR2 in MTFR2-KO cell lines and detected FUNDC1 phosphorylation; the level of FUNDC1 phosphorylation increased with the re-expression of MTFR2 (Fig. 4D). Reports have demonstrated that FUNDC1 phosphorylation inhibits mitochondrial autophagy(16). We next transfected MTFR2-KO cell lines with the phosphorylated FUNDC1 mimic FUNDC1 T18E (Rescue) and examined the autophagy status. The results showed that the ratio of LC3B-II to LC3B-I and the level of p62 were completely restored (Fig. $4 \mathrm{E})$, indicating that FUNDC1 phosphorylation was responsible for the MTFR2-mediated autophagy switch.

\section{MTFR2 confers endocrine therapy resistance to BC cells in a FUNDC1-dependent manner.}


We further investigated the mechanism by which MTFR2 alters the autophagy status. We next detected the endocrine therapy resistance ability in the rescue cell lines, and the results are shown in Fig. 5 . Cell viability and colony formation ability were completely restored in the rescue cell line (Fig. 5A, 5B, $p<$ 0.001). Flow cytometry analysis demonstrated that the apoptosis rate in the rescue cells decreased to baseline compared to that of the wild-type (WT) (Fig. 5C, p < 0.001). Apoptosis markers, such as cleaved caspase, were also reverted (Fig. 5D). Taken together, these findings suggest that MTFR2-induced autophagy is responsible for chemotherapy resistance and that this acquired resistance occurs in a FUNDC1-dependent manner.

\section{MTFR2 maintains endocrine therapy resistance in vivo.}

We demonstrated that MTFR2 promotes endocrine therapy resistance. To further uncover the biological function of this protein, we established a xenograft mouse tumor model and the results are shown in Fig. 6. The tumor volume decreased in MTFR2-KO cell lines after treatment with TMX or Let. Immunohistochemistry (IHC) analysis of MTFR2 and p-FUNDC1 levels correlated with the former results. To mimic the clinical therapeutic strategy, we applied the AAV injection assay. We injected AAV containing empty vector (NC) or specific shRNA into the tumors every three days, the results were shown in Fig. 7. The results showed that subsequent shRNA injection could also sensitize BC cells to endocrine therapy. Taken together, MTFR2 promotes the endocrine therapy resistance by directly promoting the phosphorylation of FUNDC1 and thus inhibiting the mitophagy (Fig. 8).

\section{Conclusion}

Our research demonstrate that MTFR2 is upregulated in ER + BC tumors and that MTFR2 confers endocrine therapy resistance to breast cancers through modulating autophagy flux.

\section{Discussion}

$\mathrm{BC}$ is one of the most common malignancies in women worldwide. The prognosis has improved with advances in individualized treatment. Some patients still suffer from cancer progression, such as metastasis and chemotherapy resistance. One of the most popular concepts is that dysregulation of autophagy contributes to tumorigenesis and progression of $B C(17)$. In this study, we found that MTFR2 expression was upregulated in $\mathrm{ER}+\mathrm{BC}$ patients and confers endocrine therapy resistance to $\mathrm{BC}$ cells. These effects occur by modulating mitochondrial autophagy through directly binding to FUNDC1 and promoting FUNDC1 phosphorylation. FUNDC1 is a novel factor in mitochondrial autophagy that is critical. The dysregulation of mitochondrial autophagy has been reported in many cancers and is associated with the progression of cancers such as colon, lung and gastric cancer. Generally, the total level of FUNDC1 reflects the level of mitochondrial autophagy. In this study, the total level of FUNDC1 was unchanged. Studies have demonstrated that the phosphorylation of FUNDC1 inhibits autophagic flux(18). We demonstrated that MTFR2 promotes the phosphorylation of MTFR2 and mediates 
autophagy inhibition, which is responsible for endocrine therapy resistance. Autophagy levels were reported to be upregulated in the progression of cancers(19), and our results showed that the activation of mitochondrial autophagy and an increase in autophagy influx confers endocrine therapy resistance to BC cells. We hypothesized that autophagy was enhanced because recycling was enhanced, while most recycled proteins were unimportant, and the survival substrate was not necessary.

MTFR2 plays an important functional role in mitochondria and promotes mitochondrial fission. Studies have demonstrated that MTFR2 is associated with the progression and tumorigenesis of cancers, but the function has not been thoroughly studied, and the exact mechanism is unknown. We have shown that MTFR2 could promote the invasion and proliferation of BC by switching OXPHOS to glycolysis in a HIF1a-dependent manner(14). KEGG pathway analysis results suggested that MTFR2 was responsible for endocrine therapy resistance. We next performed a series of experiments and found that MTFR2 promotes the development of endocrine therapy resistance. In the in vivo experiments, knocking out MTFR2 sensitized cancer cells to TMX and Let; however, in clinical practice, gene knockout is often unavailable, and so we next applied AAV injection assays to mimic the therapeutic strategy. The results showed that AAV injection also sensitized cancer cells to TMX and Let treatment. Taken together, these findings show that MTFR2 is a novel therapeutic target for patients with TMX and Let resistance.

\section{Methods}

\section{Patient information and clinical sample collection}

In total, 30 paired $\mathrm{ER}+\mathrm{BC}$ tissue samples and adjacent normal tissues were obtained from patients who underwent surgery at the First Affiliated Hospital of Sun Yat-sen University (Guangzhou, China) between January 2013 and December 2014. Samples before/after endocrine therapy including criterion: patients who underwent pathological sampling and receive standard endocrine therapy and breast tumor was eventually excised by surgical operation. All BC tissue samples were confirmed by pathology. This study was approved by the Ethics Committee of the First Affiliated Hospital of Sun Yatsen University and conformed to the 1964 Declaration of Helsinki and its later amendments or comparable ethical standards. Clinical samples were collected from patients after written informed consent was obtained.

\section{Quantitative real-time polymerase chain reaction (qRT-PCR)}

Total RNA was extracted with TRIzol reagent (Invitrogen, NY, USA) according to the manufacturer's protocol. The relative RNA level was normalized to $\beta$-actin, and all experiments were performed in triplicate. The primer sequences used are listed below:

MTFR2 F: 5'-GAAACTGGATCCCAATGTGAA-3' and

R: 5'-GAATAAGGTTAAGCTTCGTGCAA-3'. 


\section{Cell culture and cell transfection assay}

Human mammary cancer cell lines (T47D and MCF-7) were obtained from the American Type Culture Collection (ATCC, Manassas, VA). Cells were cultured in Dulbecco's modified Eagle's medium (DMEM) supplemented with $10 \%$ fetal bovine serum (Life Technologies, NY, USA), $1 \%$ penicillin

G, and streptomycin. CRISPR-Cas9 targeting RNA was obtained from Shanghai Generay Biotech Co., Ltd. The transfection assay was performed using Lipofectamine 3000 (Invitrogen, CA, USA) according to the manufacturer's protocol when the cells reach approximately $50-70 \%$ confluence.

\section{Immunoblotting}

Cells were washed twice with PBS and then lysed in cold RIPA buffer with protease inhibitors. Total protein $(30-60 \mu \mathrm{g})$ was separated by $12 \%$ SDS-PAGE and transferred to a nitrocellulose membrane. After being blocked with $5 \%$ nonfat milk in Tris-buffered saline containing $0.1 \%$ Tween-20 (TBST) for 1 hour at room temperature, the membranes were then incubated overnight with the corresponding primary antibodies. After being incubated with the secondary antibody, the proteins were detected using ECL (EMD Millipore, MA, USA). $\beta$-Actin was used as an internal control.

\section{CRISPR/Cas9-mediated gene knockout}

The target sequences of gRNA were designed using the online tool at http://crispr.mit.edu/. CRISPRlentivirus were harvested and used to infect cells for 4-6 hr in the incubator. After five days culture. K.O. stable cell lines were harvested.

\section{Cell proliferation assay}

Cell Counting Kit-8 (CCK-8, Dojindo, Tabaru, Japan) was used to measure cell proliferation. Cells were seeded into 96-well plates. After treatment with TMX and Let, the relative absorption of CCK-8 was measured and normalized.

\section{Colony formation assay}

To determine long-term effects on proliferation, the cells were seeded in a six-well plate. After being treated with TMX and Let, the cells were incubated for 14 days, the colonies were stained with crystal violet (Sigma-Aldrich, St. Louis, MO, USA), and the number of colonies was counted.

\section{Xenograft mouse model}


Four-week-old $B L A B / c$ nude mice were randomly divided into groups and received injections of $5 \times 10^{5}$ cells. Tumor volume was measured using a Vernier caliper. For the AAV injection assay, specific cells were injected subcutaneously, AAVs containing empty vector and specific shRNA were injected into the tumor every 3 days, and the tumor volume was measured, the shRNA sequence was previously described (14).

\section{Statistical analysis}

All data analyses were performed with SPSS 20.0 statistical software. The $\chi^{2}$ test was used to analyze the relationships between categorical variables. The differences between groups were compared by Student's $t$-tests. Cox regression and Kaplan-Meier analyses were used to analyze overall survival (OS), and $p<0.05$ was considered to be a significant difference from the control.

\section{Abbreviations}

qRT-PCR: quantitative real-time polymerase chain reaction

BC: breast cancer

MTFR2: Mitochondrial fission regulator 2

TMX: tamoxifen

Let: letrozole

K.O.: knock out

\section{Declarations}

\section{Ethics approval and consent to participate}

Studies were performed in accordance with the Declaration of Helsinki and received approval from the Ethics Committee of the First Affiliated Hospital of Sun Yat-sen University University. Clinical samples were collected from patients after written informed consent was obtained. The animal studies were approved by the Laboratory Animal Care Committee of The First Affiliated Hospital of Sun Yat-sen University.

\section{Consent for publication}

Not applicable

\section{Acknowledgments}


We thank the patients involved in this study.

\section{Competing interests}

The authors declare no competing interests.

\section{Availability}

All data in this article are available.

\section{Funding}

This article was founded by Project of National Natural Science Foundation of China (NO.32060208), General program of Guangxi Natural Science Foundation (N0.2019JJA140071), The First Batch of Highlevel Talent Scientific Research Projects of the Affiliated Hospital of Youjiang Medical University for Nationalities in 2019(Contract No. R20196307),Project of Youjiang Medical University for nationality (No.YY2019ky001).

\section{Author contribution}

JL,WML and YHL conceptualized and designed the article. ZQZ, GML, BL and FJL carried out the molecular genetic studies, established the stable cell lines and drafted the manuscript. ZPW carried out the immunoassays. GML, BL performed the statistical analysis. FJL participated in coordination and helped to draft the manuscript. All authors read and approved the final manuscript.

\section{References}

1. Ralston J, Asante K. The architects of universal health coverage. Lancet. 2019;394(10214):2071-2. doi:10.1016/S0140-6736(19)31905-1. PubMed PMID: 31818408.

2. Hortobagyi GN. Breast Cancer: 45 Years of Research and Progress. J Clin Oncol (2020):02000199. doi: 10.1200/JC0.20.00199. PubMed PMID: 32255711.

3. Crunkhorn S. Combination therapy combats aggressive breast cancer. Nat Rev Drug Discov (2020). doi: 10.1038/d41573-020-00065-5. PubMed PMID: 32249823.

4. Hong Y, Sonneville R, Wang B, Scheidt V, Meier B, Woglar A, et al LEM-3 is a midbody-tethered DNA nuclease that resolves chromatin bridges during late mitosis. Nat Commun (2018) 9(1):728. doi: 10.1038/s41467-018-03135-w. PubMed PMID: 29463814.

5. Takeshita T, Yamamoto Y, Yamamoto-lbusuki M, Tomiguchi M, Sueta A, Murakami K, et al Clinical significance of plasma cell-free DNA mutations in PIK3CA, AKT1, and ESR1 gene according to 
treatment lines in ER-positive breast cancer. Mol Cancer(2018) 17(1):67. doi: 10.1186/s12943-0180808-y. PubMed PMID: 29482551.

6. Sokol ES, Feng YX, Jin DX, Basudan A, Lee AV, Atkinson JM, et al. Loss of function of NF1 is a mechanism of acquired resistance to endocrine therapy in lobular breast cancer. Ann Oncol. 2019;30(1):115-23. doi:10.1093/annonc/mdy497. PubMed PMID: 30423024.

7. Liu GY, Sabatini DM. mTOR at the nexus of nutrition, growth, ageing and disease. Nat Rev Mol Cell Biol (2020) 21(4):183-203. doi: 10.1038/s41580-019-0199-y. PubMed PMID: 31937935.

8. Tsugawa H, Mori H, Matsuzaki J, Sato A, Saito Y, Imoto M, et al. CAPZA1 determines the risk of gastric carcinogenesis by inhibiting Helicobacter pylori CagA-degraded autophagy. Autophagy. 2019;15(2):242-58. doi: 10.1080/15548627.2018.1515530. PubMed PMID: 30176157.

9. Liu M, Sun T, Li N, Peng J, Fu D, Li W, et al BRG1 attenuates colonic inflammation and tumorigenesis through autophagy-dependent oxidative stress sequestration. Nat Commun (2019) 10(1):4614. doi: 10.1038/s41467-019-12573-z. PubMed PMID: 31601814.

10. Qu F, Wang P, Zhang K, Shi Y, Li Y, Li C, et al. Manipulation of Mitophagy by "All-in-One" nanosensitizer augments sonodynamic glioma therapy. Autophagy (2019):1-23. doi: 10.1080/15548627.2019.1687210. PubMed PMID: 31674265.

11. Marsh T, Debnath J. Autophagy Suppresses Breast Cancer Metastasis By Degrading NBR1. Autophagy (2020). doi: 10.1080/15548627.2020.1753001. PubMed PMID: 32267786.

12. Lin JZ, Wang WW, Hu TT, Zhu GY, Li LN, Zhang CY, et al. FOXM1 contributes to docetaxel resistance in castration-resistant prostate cancer by inducing AMPK/mTOR-mediated autophagy. Cancer Lett. 2020;469:481-9. doi: 10.1016/j.canlet.2019.11.014. PubMed PMID: 31738958.

13. Urbanska K, Orzechowski A. Unappreciated Role of LDHA and LDHB to Control Apoptosis and Autophagy in Tumor Cells. Int J Mol Sci (2019) 20(9). doi:10.3390/ijms20092085. PubMed PMID: 31035592.

14. Lu G, Lai Y, Wang T, Lin W, Lu J, Ma Y, et al. Mitochondrial fission regulator 2 (MTFR2) promotes growth, migration, invasion and tumour progression in breast cancer cells. Aging. 2019;11(22):10203-19. doi:10.18632/aging.102442. PubMed PMID: 31740625.

15. Lin Z, Niu Y, Wan A, Chen D, Liang H, Chen $X$, et al. RNA m(6) A methylation regulates sorafenib resistance in liver cancer through FOXO3-mediated autophagy. Embo J (2020):e103181. doi:10.15252/embj.2019103181. PubMed PMID: 32368828.

16. Kuang Y, Ma K, Zhou C, Ding P, Zhu Y, Chen Q, et al. Structural basis for the phosphorylation of FUNDC1 LIR as a molecular switch of mitophagy. Autophagy. 2016;12(12):2363-73. doi:10.1080/15548627.2016.1238552. PubMed PMID: 27653272.

17. Martinez-Outschoorn UE, Peiris-Pages M, Pestell RG, Sotgia F, Lisanti MP. Cancer metabolism: a therapeutic perspective. Nat Rev Clin Oncol. 2017;14(1):11-31. doi:10.1038/nrclinonc.2016.60. PubMed PMID: 27141887.

18. Zhou H, Zhu P, Wang J, Zhu H, Ren J, Chen Y. Pathogenesis of cardiac ischemia reperfusion injury is associated with CK2alpha-disturbed mitochondrial homeostasis via suppression of FUNDC1-related 
mitophagy. Cell Death Differ. 2018;25(6):1080-93. doi:10.1038/s41418-018-0086-7. PubMed PMID: 29540794.

19. Wang ZH, Wang JH, Wang KQ, Zhou Y, Wang J. LncRNA FEZF1-AS1 promoted chemoresistance, autophagy and epithelial-mesenchymal transition (EMT) through regulation of miR-25-3p/ITGB8 axis in prostate cancer. Eur Rev Med Pharmacol Sci. 2020;24(5):2281-93. doi: 10.26355/eurrev_202003_20494. PubMed PMID: 32196579.

\section{Figures}
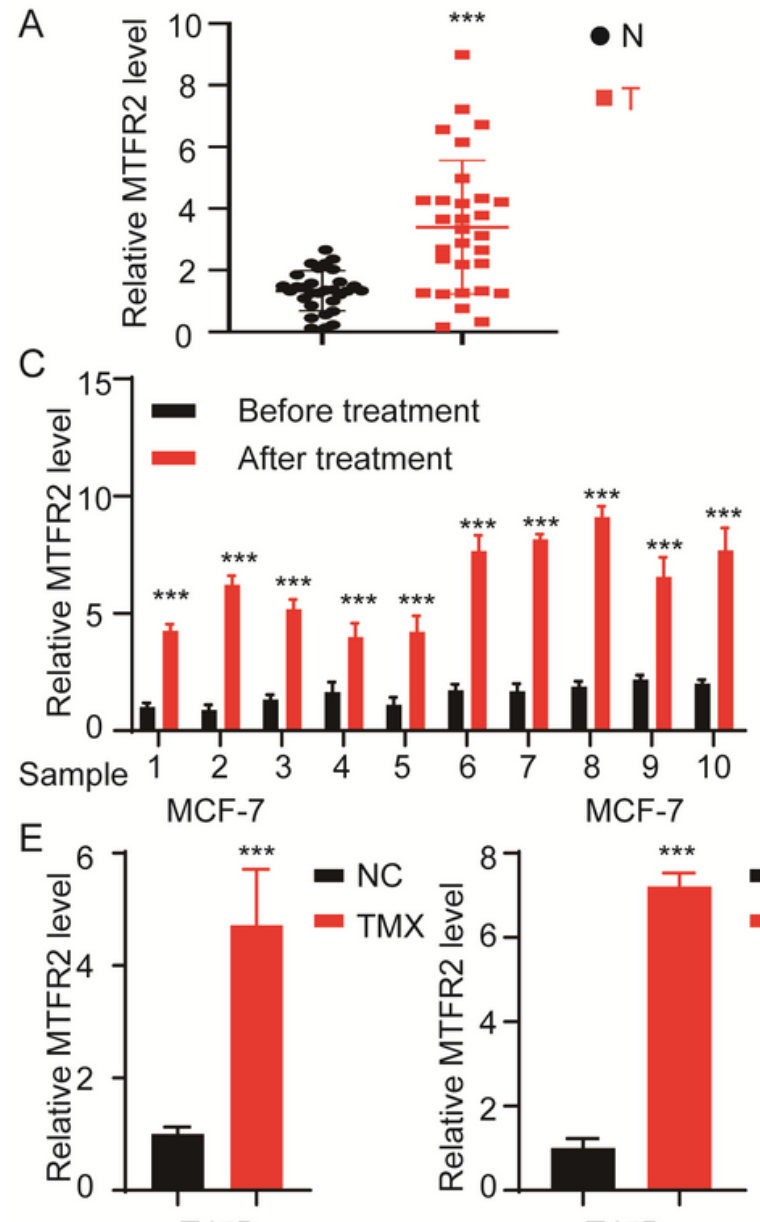

T47D

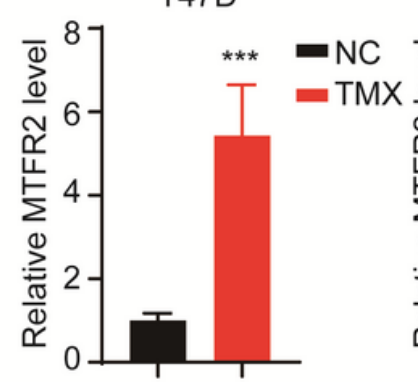

$\mathrm{F}$

MCF-7

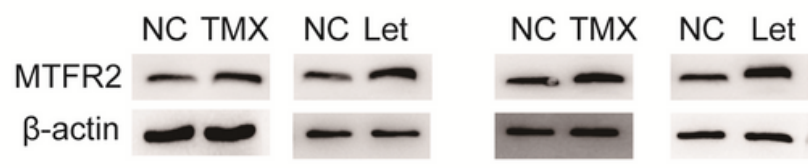

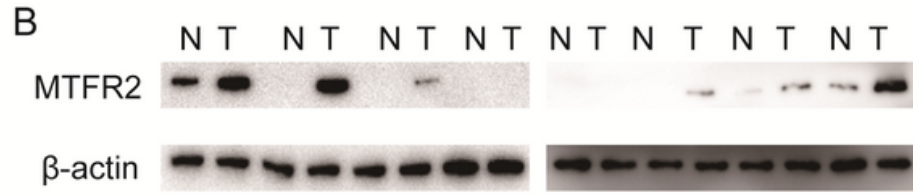

D Sample $\frac{1}{\square \square} \frac{2}{\square \square} \frac{3}{\square \square} \frac{4}{\square} \frac{5}{\square}$

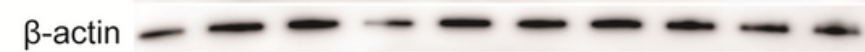

Sample $\frac{1}{\square} \frac{2}{\square} \frac{3}{\square \square} \frac{4}{\square} \frac{5}{\square}$

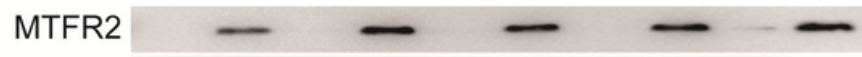

- Before treatment

- After treatment

G

Pyrimidine Metabolism

- Let

Proteasome

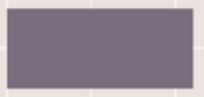

P53 Signaling Pathway

Apoptosis

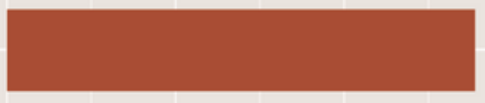

Mismatch Repair

DNA Replication

Methionine Metabolism

Cell Cycle
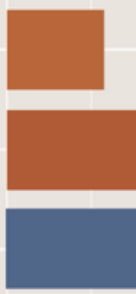

-lg.P

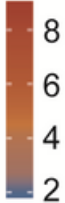

\section{Basal Excision Repair-}

tRNA Biosynthesis

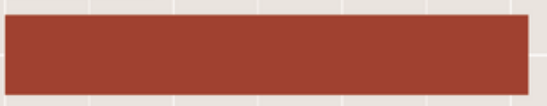




\section{Figure 1}

MTFR2 was upregulated in $E R+B C$ and was correlated with endocrine therapy resistance. A: The relative RNA level of MTFR2 of the in-house database(Student's two-tailed paired t-test; ${ }^{* \star \star} p<0.001, n=30$ ). B: 8 tumors and paired normal tissues were randomly selected from our in-house cohort, the protein level of MTFR2 was detected. C: 10 paired patients before/after anti endocrine -therapy were enrolled, the samples were collected, the relative RNA level of MTFR2 was detected (Student's two-tailed paired t-test; $\star \star \star p<0.001)$. D: The protein level of MTFR2 in the samples described in C. E: The relative RNA level of MTFR2 in MCF-7 or T47D cells treated with TMX or Let (Student's two-tailed paired t-test; $\star \star \star ~ p<0.001)$. F: Immunoblot showing MTFR2 expression in MCF-7 or T47D cells treated with TMX or Let. G: KEGG pathway analysis. 
A
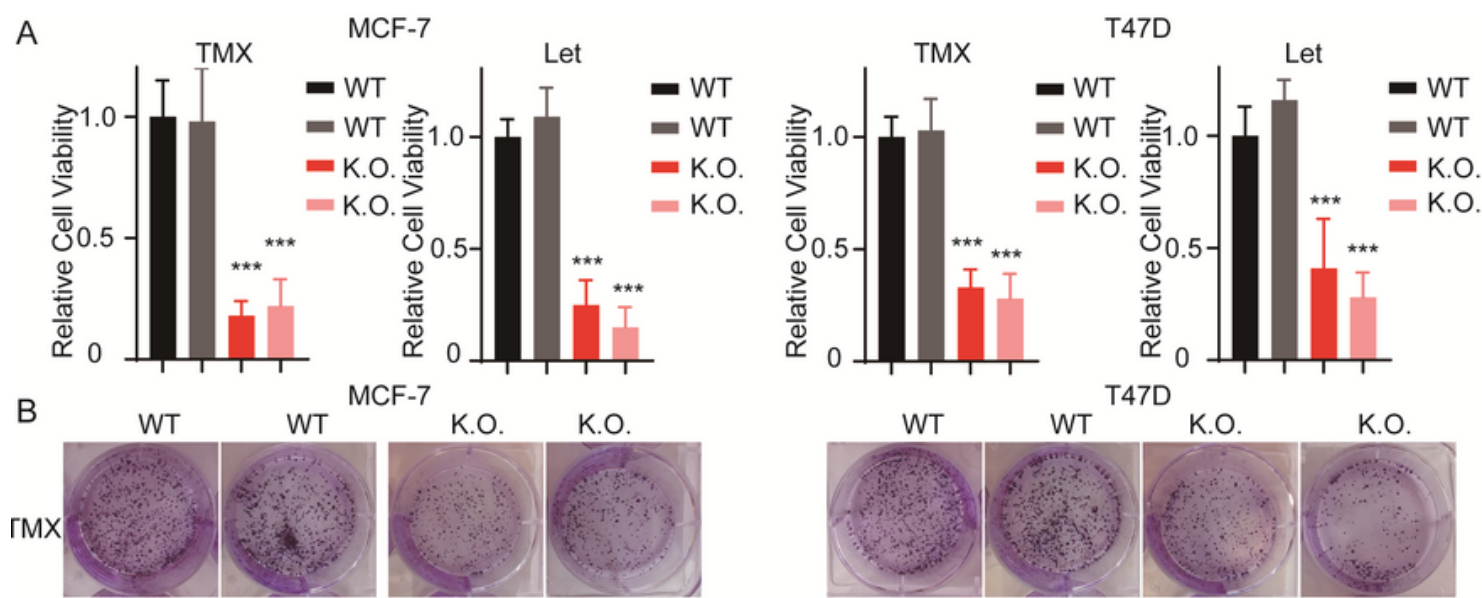

Let
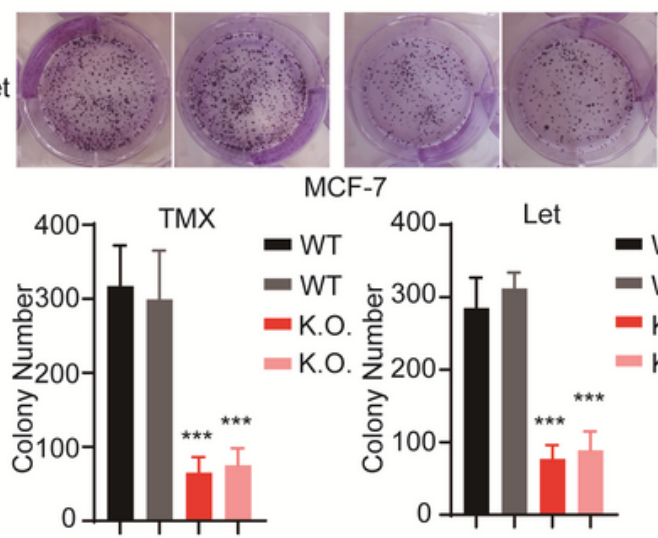

MCF-7

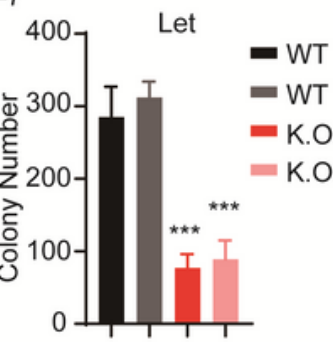

MCF-7
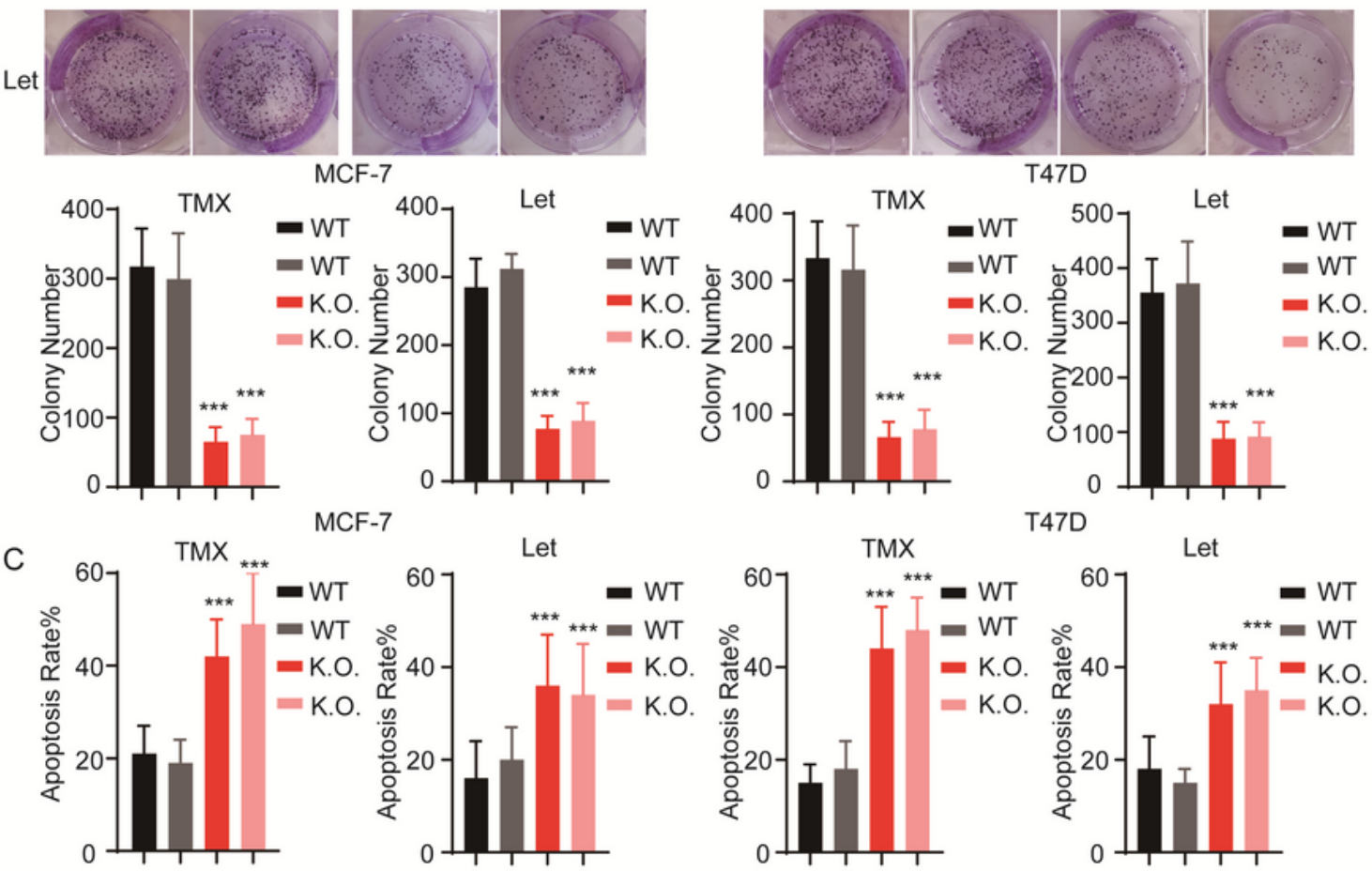

D

MCF-7

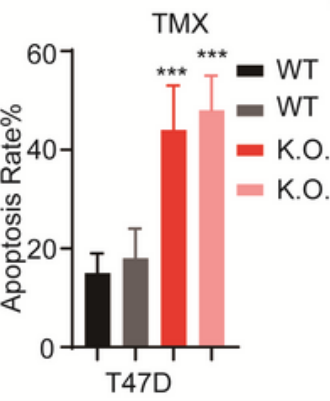

Let

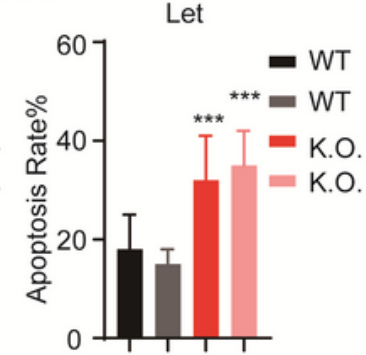

TMX

Let

TMX

Let

WT WT K.O.K.O. WT WT K.O.K.O

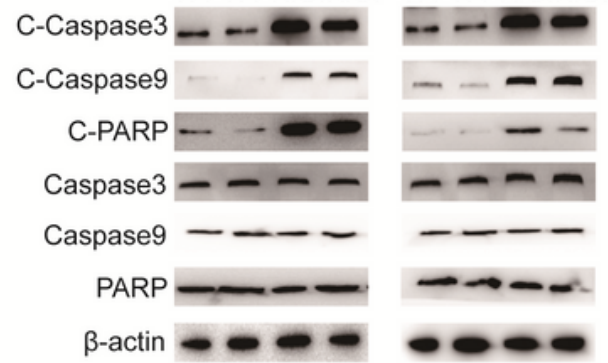

WT WT K.O.K.O. WT WT K.O.K.O.

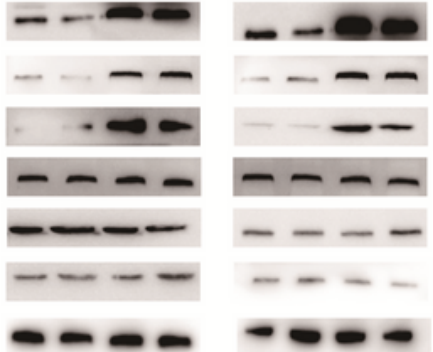

Figure 2

MTFR2 promotes the endocrine therapy resistance of BC cells. A: We established stable MTFR2 knocking out cell lines of MCF-7 and T47D. Cells were then treated with TMX or Let and then subjected to CCK-8 assay. The relative cell viability of the cell lines was detected. (Student's two-tailed paired t-test; *ᄎ* p <0.001). B: Upper: Representative image of colony formation assay of different cell lines with indicated modification. Lower: The colony number of different cell lines. (Student's two-tailed paired t-test; *** 
$\mathrm{p}$ <0.001). C: Apoptosis rate was detected by flow cytometry (Student's two one-tailed paired t-test; * p 0.001). D: Western blot analysis of apoptosis markers. C-caspase3, C-caspase9 and C-PARP was short for cleaved caspase3, cleaved caspase9 and cleaved PAPR, respectively.

A

MCF-7

TMX

WT WT K.O. K.O. WT WT K.O. K.O.

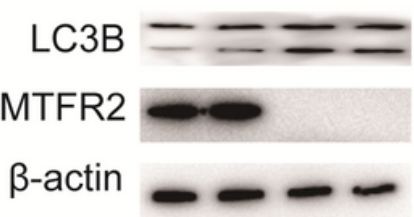

T47D

TMX

Let

WT WT K.O. K.O. WT WT K.O. K.O.

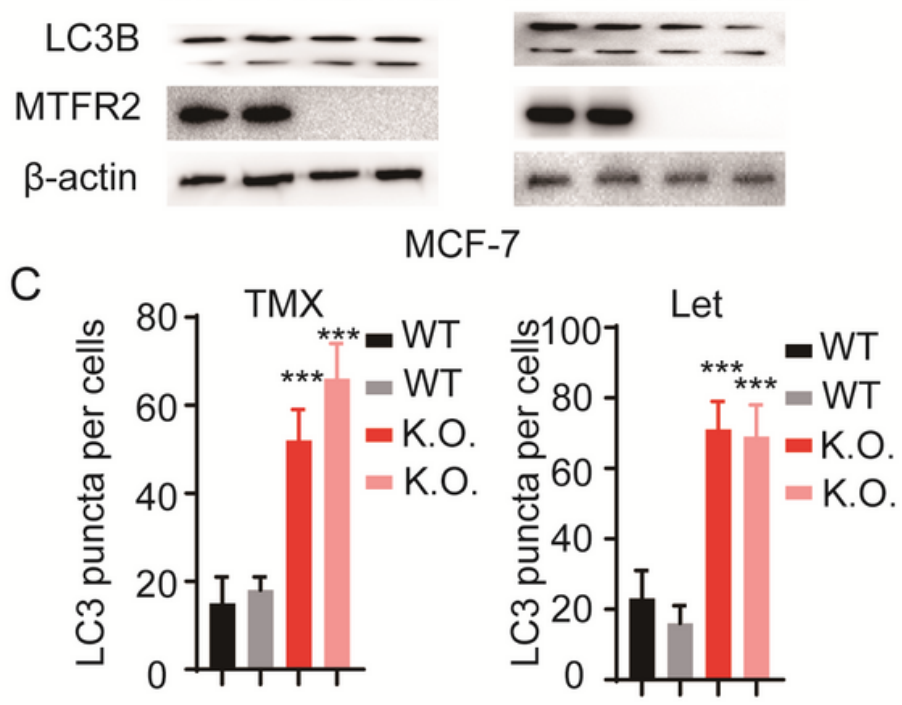

T47D
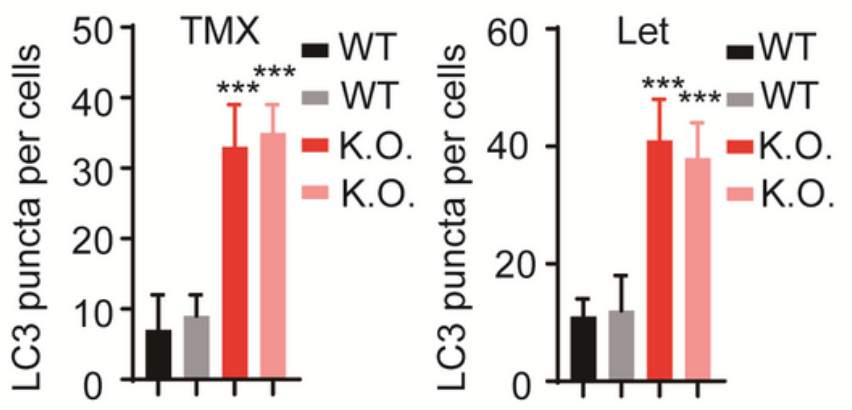

$\mathrm{B}$
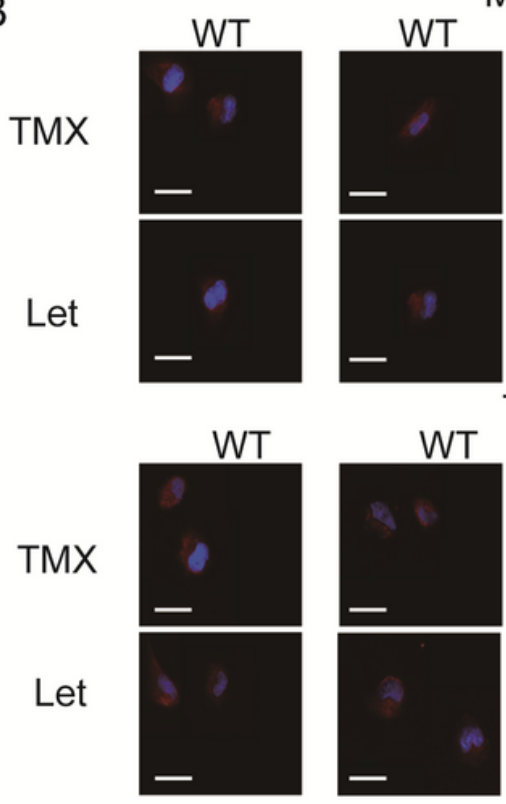

T47D
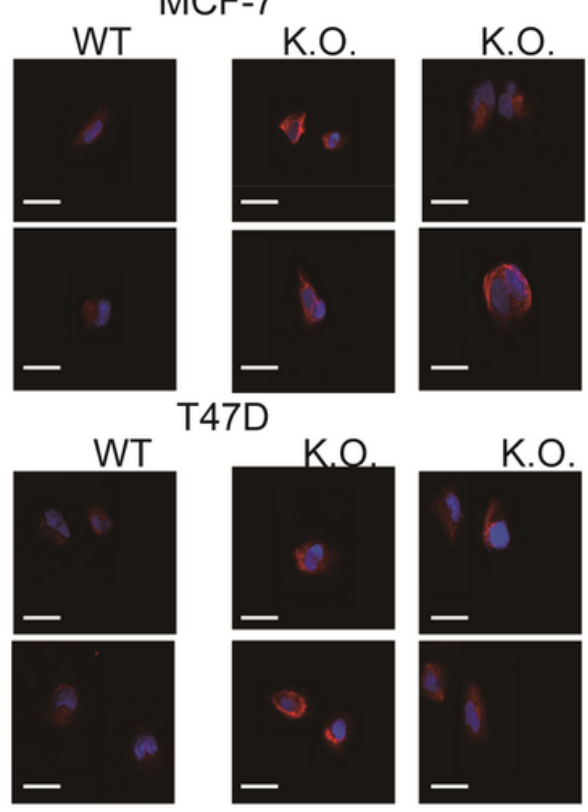

D

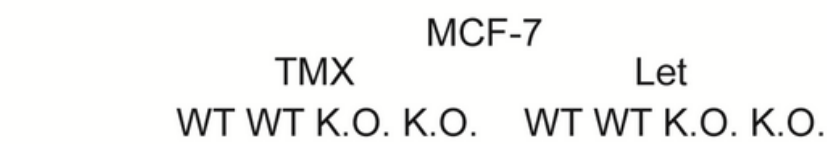

p62

$\beta$-actin

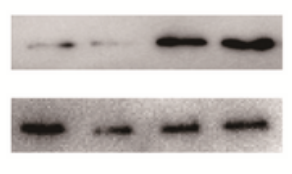

T47D

TMX WT WT K.O. K.O. WT WT K.O. K.O. p62

$\beta$-actin
Let
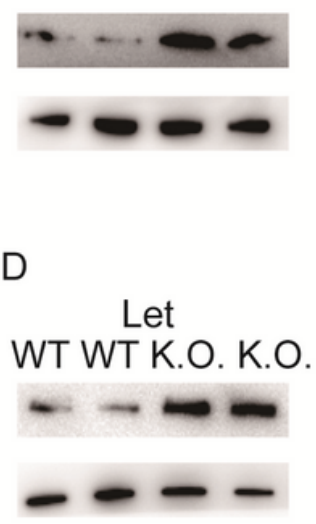

\section{Figure 3}

MTFR2 knockout enhances the autophagic flux of BC. A: Immunoblot analysis of LC3B and MTFR2 in different cell lines with indicated modification. B: Immunofluorescence of LC3B in the different cell lines with 400x magnification, scale $20 \mu \mathrm{m}$. C: Statistical analysis of the number of LC3 puncta per cell of different cell lines. (Student's two one-tailed paired t-test; $\left.{ }^{* \star} p<0.001\right) \mathrm{D}$ : Immunoblot analysis of $p 62$. 

A
MCF-7
T47D
Input $\frac{\mathrm{IP}: \text { Flag }}{\operatorname{IgG} \text { Flag }}$
IP:Flag
$\mathrm{HA}$
Input IgG Flag
B
C

\section{MCF-7}
TMX
Let
D

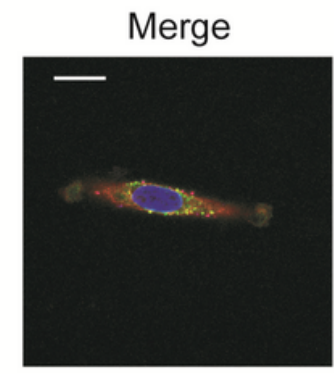

MTFR2

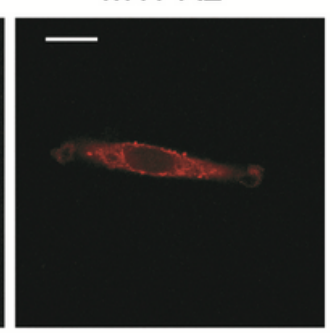

FUNDC1

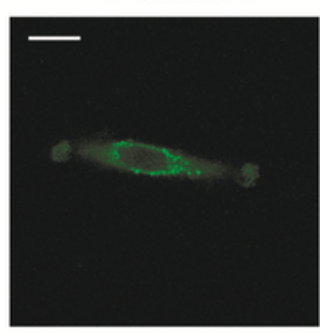

WT WT K.O. K.O. WT WT K.O. K.O.

\section{p-FUNDC1 \\ FUNDC1 \\ $\beta$-actin}

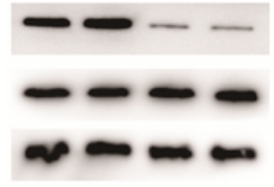

T47D

TMX

WT WT K.O. K.O. WT WT K.O. K.O.

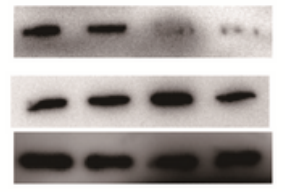

Let

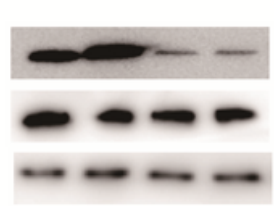

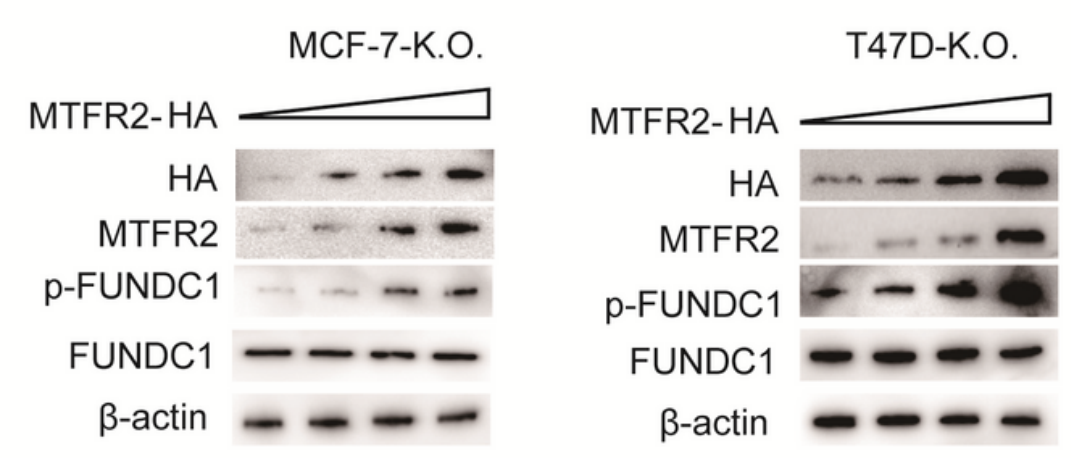

$E$

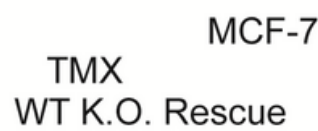

p-FUNDC1

FUNDC1

LC3B

P62

MTFR2

$\beta$-actin

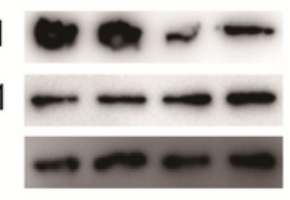

Let WT K.O. Rescue

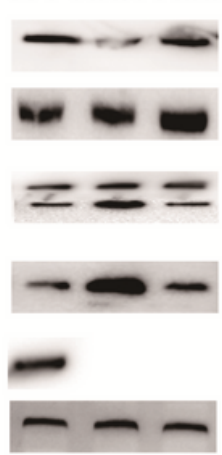

T47D

TMX WT K.O. Rescue

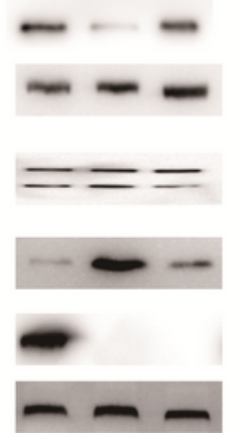

Let WT K.O. Rescue

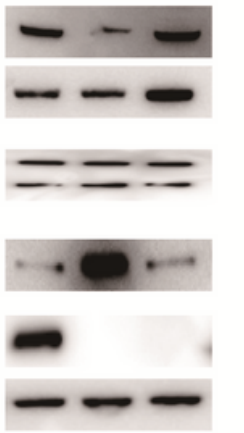

Figure 4

MTFR2 directly binds with FUNDC1 and promotes FUNDC1 phosphorylation and downstream autophagy. A: IP of HA-MTFR2 and Flag-FUNDC1 in MCF-7 and T47D cells. B: Immunofluorescence of MTFR2 and FUNDC1 in MCF-7 cells, scale $20 \mu \mathrm{m}$. C: Immunoblot showing the p-FUNDC1/FUNDC1 ratio in different cell lines. D: Immunoblot showing the p-FUNDC1/FUNDC1 ratio and MTFR2 expression in MTFR2-K.O. cell lines with MTFR2 re-expression. E: Western blot showing the p-FUNDC1/FUNDC1 ratio and MTFR2, LC3B and p62 expression in WT, K.O. and Rescue (K.O. cells transfected with FUNDC1 T18E) cell lines. 
A
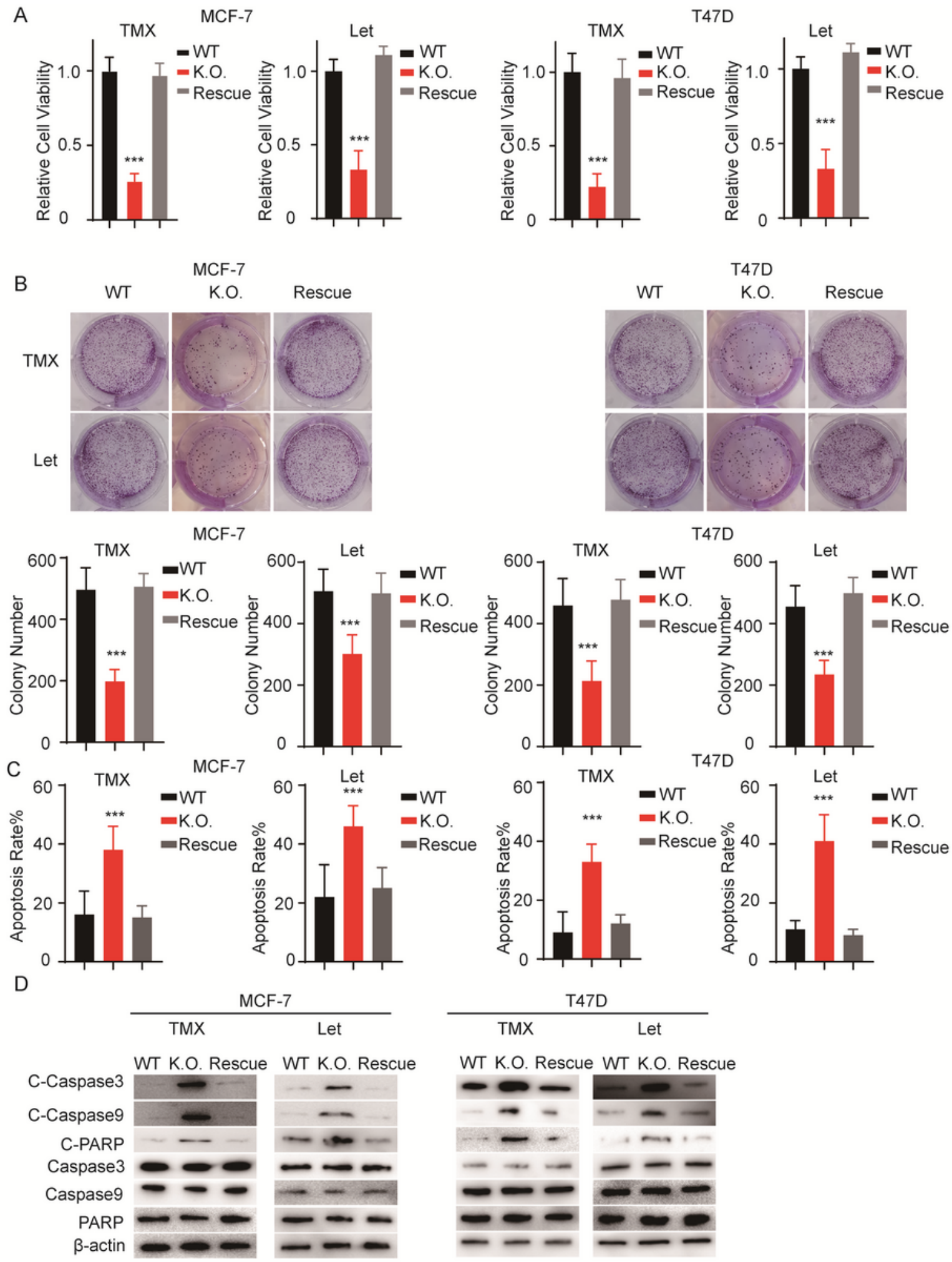

Figure 5

MTFR2 confers endocrine therapy resistance to BC cells in a FUNDC1-dependent manner. A: The relative cell viability of the cell lines treated with TMX or Let with indicated modification. (Student's two-tailed paired t-test; $\left.{ }^{* \star} p<0.001\right)$. B: Upper: Representative image of colony formation assay results in different cell lines. Lower: The colony number of different cell lines. (Student's two-tailed paired t-test; $* \star \star p<0.001$ ). C: The apoptosis rate was detected by flow cytometry (Student's two one-tailed paired t-test; * $p<0.001$ ). 
D: Immunoblot analysis of apoptosis markers. C-caspase3, C-caspase9 and C-PARP was short for cleaved caspase3, cleaved caspase9 and cleaved PAPR, respectively.

A

MCF-7

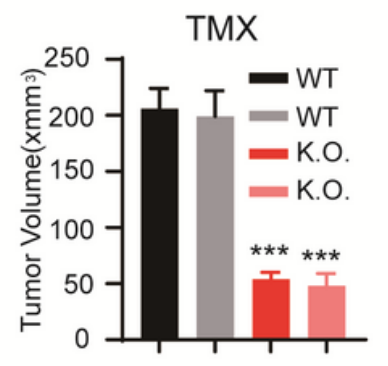

C

WT

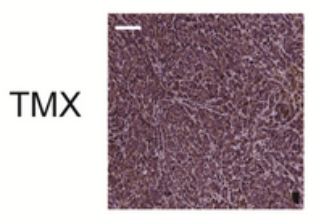

Let

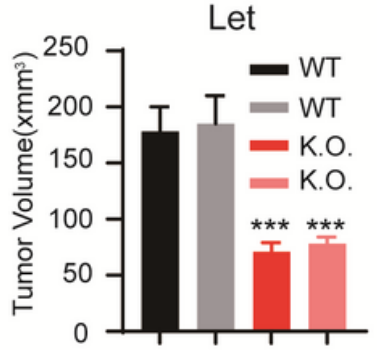

MCF-7
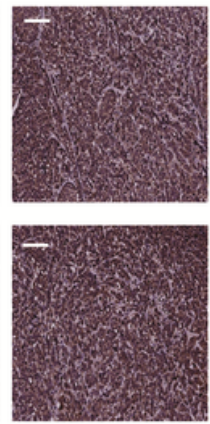

K.O.

K.O.
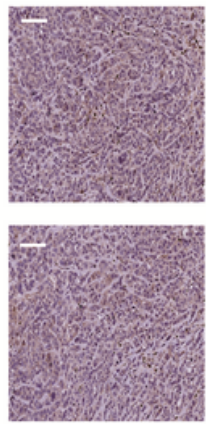

$\mathrm{B}$
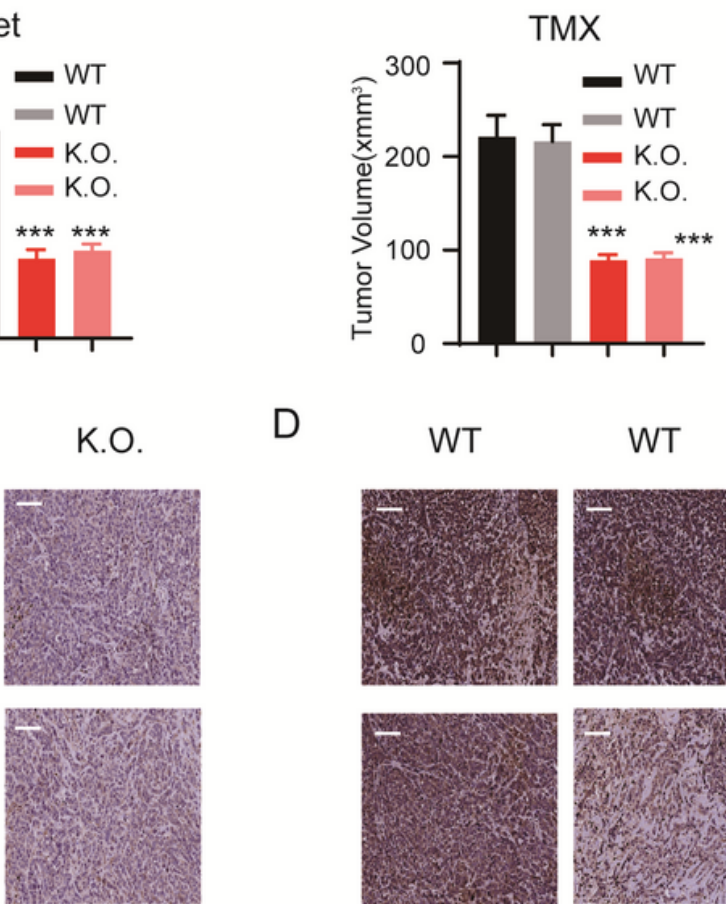

WT

T47D

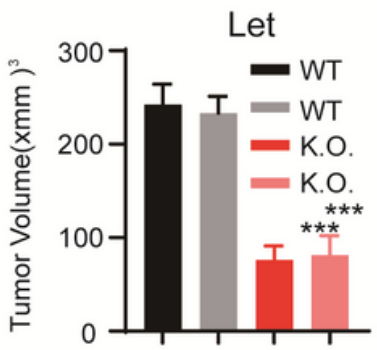

T47D

WT
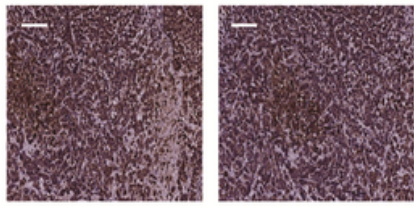

K.O.

K.O.

Figure 6

MTFR2 promotes endocrine therapy resistance in vivo. A-B: BC cells with indicated modification were planted to establish Xenograft mouse model. The tumor volume was detected (Student's two one-tailed paired t-test; * $p<0.001)$. C-D: The IHC of $p$-FUNDC1 was detected in the Xenograft mouse model with indicated modifications with 200x magnification, scale $200 \mu \mathrm{m}$. 
A

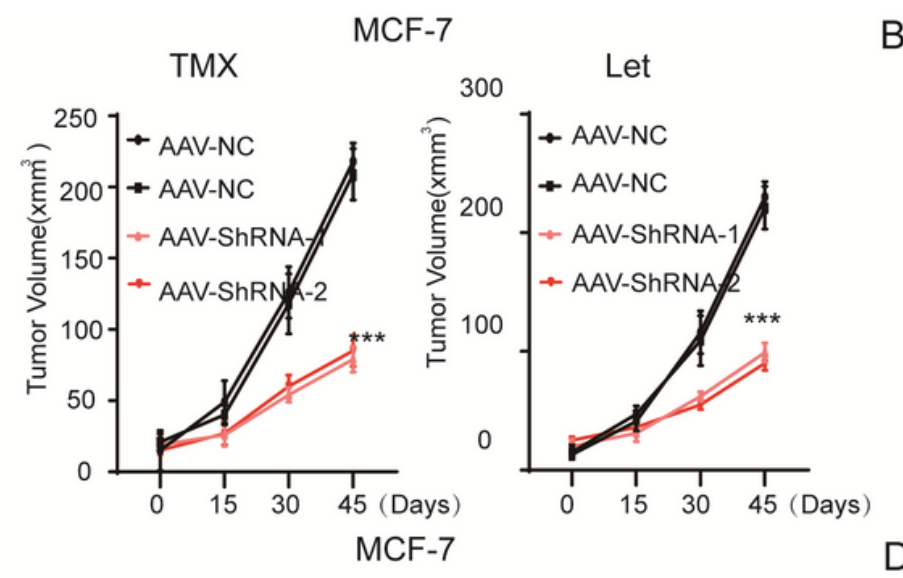

C

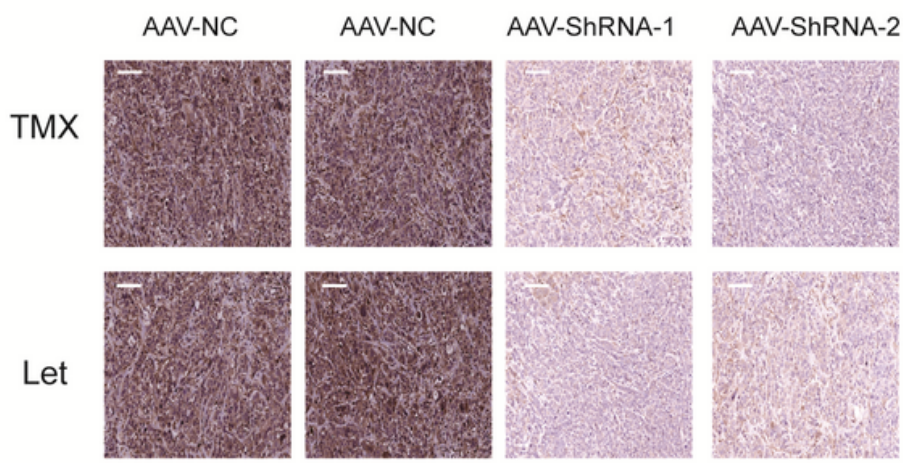

B

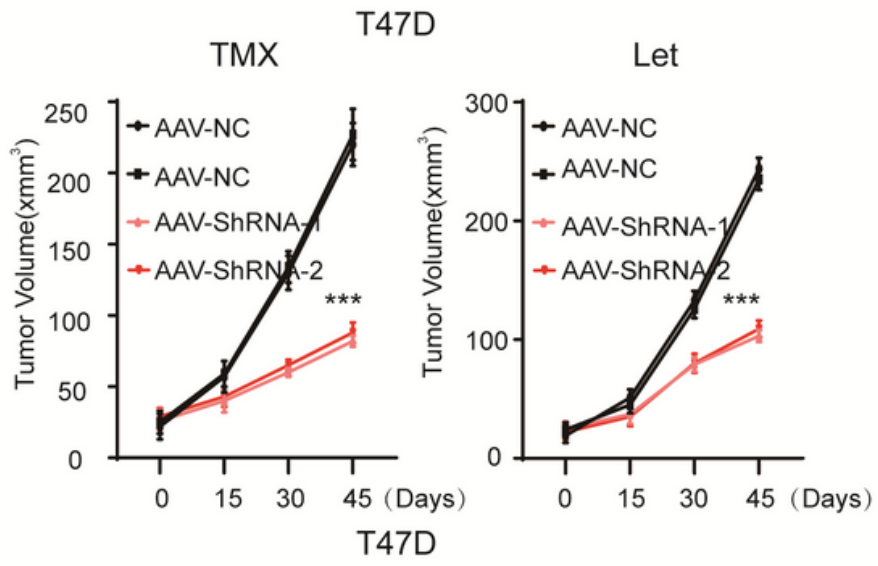

AAV-NC
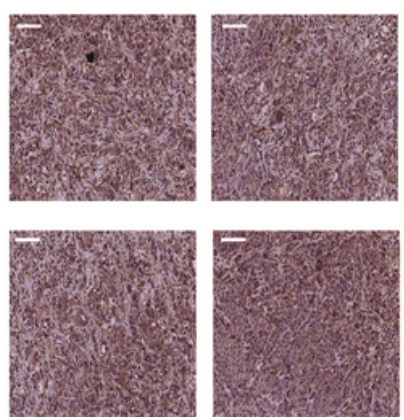
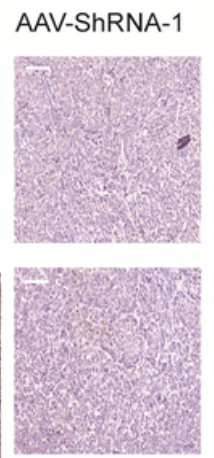

AAV-ShRNA-2
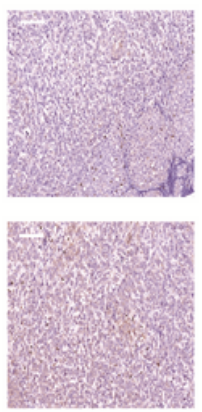

Figure 7

MTFR2 was a potential therapy target to overcome endocrine therapy resistance in vivo. A-B: To mimic the clinical application, AAV containing NC and specific shRNA targeting MTFR2 was injected to tumors every 3 days. The tumor volume was detected every 15 days, (Student's two one-tailed paired t-test; * p < 0.001). C-D: The IHC of p-FUNDC1 was detected at the end point of each cell line with 200x magnification, scale $200 \mu \mathrm{m}$.

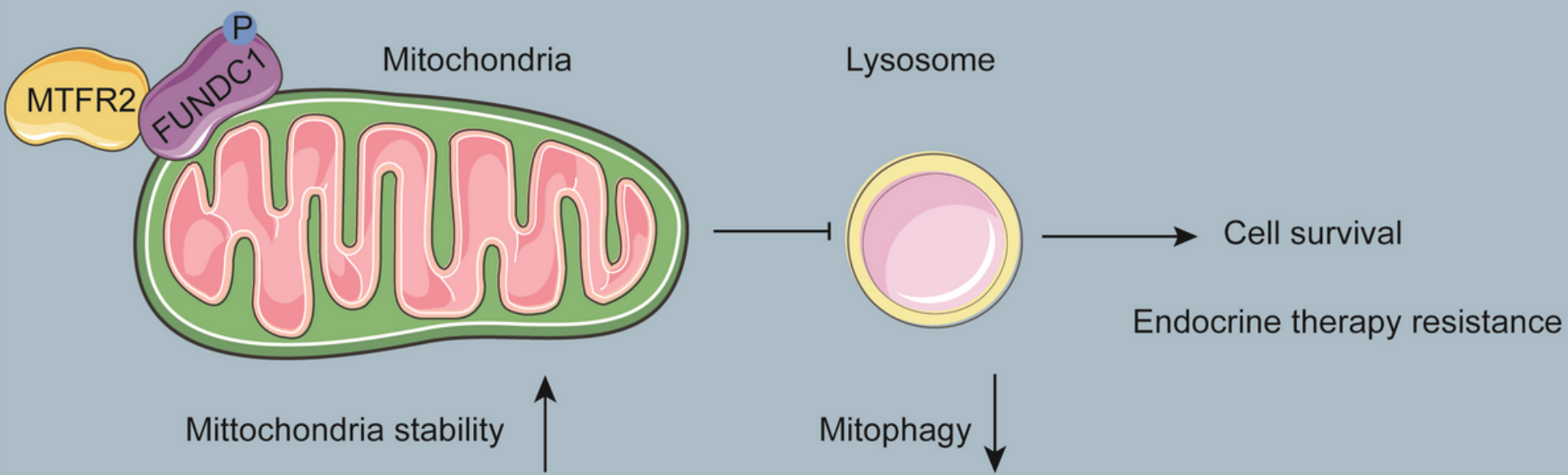

\section{Cytoplasma}

\section{Figure 8}


The graphic summary of the mechanism of MTFR2 on the endocrine therapy resistance. 